\title{
Confidence bands for percentile residual lifetimes
}

\author{
Sándor Csörgö* and László Viharos \\ Department of Statistics, University of Michigan, Ann Arbor, MI 48109-1027, USA, and Bolyai \\ Institute, University of Szeged, H-6720 Szeged, Hungary
}

Keceived 8 November 1988; revised manuscript received 26 October 1989

Recommended by $\mathbf{J}$. Koronacki

\begin{abstract}
The presently available asymptotically distribution-free confidence bands for the percentile residual lifetime function are formed. Two types of these contain theoretically unknown constants which we determine by Monte Carlo simulation. The other two types of bands are based on the bootstrap. The four types of bands are compared in an example of estimating the median residual duration time of a British strike.
\end{abstract}

\section{The bands}

Let $X \geq 0$ be a random lifetime, let $F$ denote its distribution function with support $\left(0, T_{F}\right)$, where $T_{F}=\inf \{t>0: F(t)=1\} \leq \infty$, and corresponding quantile function $Q(x)-F^{-1}(x)=\inf \{t>0: F(t) \geq x\}, 0<x<1, Q(0)=0, Q(1)-T_{F}$. Let $0<p<1$ be any fixed number. The $(1-p)$-percentile residual lifetime at $t>0$ is defined to be

$$
R^{(p)}(t)=Q(1-p(1-F(t)))-t
$$

Indeed, for any $t \geq 0$ and $0<p<1$,

$$
R^{(p)}(t)=\inf \{x>0: P\{X \leq x+t \mid X>t\} \geq 1-p\}
$$

is the $(1-p)$-percentile additional time to failure, given no failure by time $t$.

Beginning with Schmittlein and Morrison (1981), many papers suggest potential advantages of using the median residual life-time $R^{(1 / 2)}(t), t \geq 0$, instead of the more frequently used mean residual lifetime and, more generally, of using percentile residual lifetimes. The basic references are in Csörgö and Csörgö (1987) who initiate a large sample theory for the estimation of general percentile residual lifetime functions $R^{(p)}(t), t \geq 0,0<p<1$. The aim of the present note is to produce readily applicable simultaneous confidence bands for the function $R^{(p)}(t), t \geq 0$, where $p$ is

\footnotetext{
* Supported in part by the Hungarian National Foundation for Scientific Research, Grants $1808 / 86$ and $457 / 88$.
} 
fixed, and to make some comparisons among the available four types of bands on an interesting example.

Let $X_{1}, \ldots, X_{n}$ be independent observations on $X$ with corresponding order statistics $X_{1, n} \leq \cdots \leq X_{n, n}$, and consider the sample distribution and quantile functions $F_{n}(t)=n^{-1} \#\left\{1 \leq k \leq n: X_{k} \leq t\right\}, t \geq 0$, and $Q_{n}(x)=X_{k, n}$ if $(k-1) / n<x \leq k / n$, $k=1, \ldots, n ; Q_{n}(0)=X_{1, n}$. The sample analogue of $R^{(n)}(t)$ is then

$$
R_{n}^{(p)}(t)=Q_{n}\left(1-p\left(1-F_{n}(t)\right)\right)-t .
$$

For any $\lambda>0$, introduce the intervals

$$
\begin{aligned}
& A_{n}^{(p)}(\lambda ; t)=\left[R_{n}^{(p)}(t)-\frac{\lambda}{\sqrt{n} f_{n}\left(R_{n}^{(p)}(t)+t\right)}, R_{n}^{(p)}(t)+\frac{\lambda}{\sqrt{n} f_{n}\left(R_{n}^{(p)}(t)+t\right)}\right], \\
& B_{n}^{(p)}(\lambda ; t)=\left[Q_{n}\left(1-p\left(1-F_{n}(t)\right)-\frac{\lambda}{\sqrt{n}}\right), Q_{n}\left(1-p\left(1-F_{n}(t)\right)+\frac{\lambda}{\sqrt{n}}\right)\right],
\end{aligned}
$$

where, in the first one, $f_{n}$ is an estimator of the density function $f=F^{\prime}$, the existence of which will be assumed. Also consider a bootstrap sample $\tilde{X}_{1}, \ldots, \tilde{X}_{n}$, with the corresponding $\tilde{F}_{n}, \tilde{Q}_{n}$ and $\bar{R}_{n}^{(p)}$, drawn with replacement from the original sample $X_{1}, \ldots, X_{n}$, and, for a given $0<\alpha<1$, form the intervals

$$
\begin{aligned}
& C_{n}^{(p)}(\alpha ; t)=\left[R_{n}^{(p)}(t)-\frac{c_{n}(\alpha, p, T)}{\sqrt{n}}, R_{n}^{(p)}(t)+\frac{c_{n}(\alpha, p, T)}{\sqrt{n}}\right], \\
& D_{n}^{(p)}(\alpha ; t)=\left[R^{(p)}(t)-\frac{d_{n}(\alpha, p, T)}{\sqrt{n}} \sigma_{n}(t), R_{n}^{(p)}(t)+\frac{d_{n}(\alpha, p, t)}{\sqrt{n}} \sigma_{n}(t)\right],
\end{aligned}
$$

where $\sigma_{n}(t)=\left(p(1-p)\left(1-F_{n}(t)\right)\right)^{1 / 2} / f_{n}\left(R_{n}^{(p)}(t)+t\right)$ and, for some $T>0$,

$$
\begin{aligned}
& c_{n}(\alpha, p, T)=\inf \left\{y>0: P\left\{\sup _{0 \leq t \leq T} \sqrt{n}\left|\tilde{R}_{n}^{(p)}(t)-R_{n}^{(p)}(t)\right| \geq y \mid X_{1}, \ldots, X_{n}\right\} \geq 1-\alpha\right\}, \\
& d_{n}(\alpha, p, T)=\inf \left\{y>0: P\left\{\sup _{0 \leq t \leq T} \sqrt{n}\left|\frac{\tilde{R}_{n}^{(p)}(t)-R_{n}^{(p)}(t)}{\sigma_{n}(t)}\right| \geq y \mid X_{1}, \ldots, X_{n}\right\} \geq 1-\alpha\right\} .
\end{aligned}
$$

Let $B(x), 0 \leq x \leq 1$, denote a Brownian bridge and finally introduce the notation

$$
V(p)=\sup _{Q(1-p) \leq x<T_{F}} \frac{F(x)(1-F(x))\left|f^{\prime}(x)\right|}{f^{2}(x)} .
$$

Our band statements are contained in the following:

Theorem. Let $0<p<1$ and $0<\alpha<1$ be fixed, set $\delta_{n}=K n^{-1} \log \log n$ with some constant $K>0$, and let $\varepsilon_{n}$ be any sequence of positive numbers such that $\varepsilon_{n} \rightarrow 0$ and $\sqrt{n} \varepsilon_{n} \rightarrow \infty$ as $n \rightarrow \infty$.

(i) Assume that $F$ is twice differentiable on $\left(Q(1-p), T_{F}\right), f$ is positive on $\left(Q(1-p), T_{F}\right)$, and $V(p)<\infty$. If $f_{n}(\cdot)$ is a uniformly consistent estimator of $f(\cdot)$ on 
$\left(Q(1-p), T_{F}\right)$, then

$$
\lim _{n \rightarrow \infty} P\left\{R^{(p)}(t) \in A_{n}^{(p)}(\lambda(p, \alpha) ; t), 0 \leq t \leq Q_{n}\left(1-\delta_{n}\right)\right\}=1-\alpha,
$$

where $\lambda(p, \alpha)>0$ is the unique value of $\lambda$ for which

$$
P\left\{\sup _{0 \leq x \leq 1}|B(1-p(1-x))-p B(x)| \leq \lambda\right\}=1-\alpha .
$$

(ii) Assume that $F$ is twice differentiable on $\left(Q(1-p), T_{F}\right), f$ is positive on $\left(Q(1-p), T_{F}\right)$, and $V(p)<\infty$. Then

$$
\lim _{n \rightarrow \infty} P\left\{R^{(p)}(t) \in B_{n}^{(p)}(\lambda(p, \alpha) ; t), 0 \leq t \leq Q_{n}\left(1-\varepsilon_{n}\right)\right\}=1-\alpha,
$$

where $\lambda(p, \alpha)$ is as in part (i).

(iii) Assume that $f$ is positive and continuous on $(Q(1-p)-\varepsilon$, $Q(1-p(1-F(T)))+\varepsilon)$, where $\varepsilon$ is any small positive number and $T<T_{F}$. Then

$$
\lim _{n \rightarrow \infty} P\left\{R^{(p)}(t) \in C_{n}^{(p)}(\alpha ; t), 0 \leq t \leq T\right\}=1-\alpha .
$$

(iv) Assume the conditions of part (iii). If $f_{n}(\cdot)$ is a uniformly consistent estimator of $f(\cdot)$ on $(Q(1-p)-\varepsilon, Q(1-F(T))+\varepsilon)$, then

$$
\lim _{n \rightarrow \infty} P\left\{R^{(p)}(t) \in D_{n}^{(p)}(\alpha ; t), 0 \leq t \leq T\right\}=1-\alpha
$$

Part (i) follows from a modified version of Theorem 4 (given without proof) in Csörgő and Csörgő (1987) with a very lengthy proof given by Viharos (1988). Part (ii) is formulated as a part of Theorem 5 in Csörgő and Csörgô (1987) without proof. Its proof is again in Viharos (1988). These two proofs use standard strong approximation techniques established for the general quantile process. Parts (iii) and (iv) are contained in Example 7 of S. Csörgö and Mason (1989), with the proofs indicated there spelled out in detail in Viharos (1988).

The asymptotically distribution-free bands in (i) and (ii) are not applicable as they stand since they involve the constant $\lambda(p, \alpha)$ expressed through a theoretically unknown distribution. In the next section we report on an extensive Monte Carlo simulation study to determine this unknown distribution and tabulate $\lambda(p, \alpha)$ for various values of $\alpha$ and $p$. By this study the bands in (i) and (ii) become readily applicable once and for all.

The band in (ii) is particularly attractive, in principle at least, since it does not involve any density estimation. An unpleasant feature of the bands in (i) and (ii) is the presence of the sequences $\delta_{n}$ and $\varepsilon_{n}$. On the other hand, they appear to be valid almost on the whole support, this is why the strong regularity conditions are needed here. While validity on the whole support may seem desirable at first glance, we shall see in the example of Section 3 that it can also be the cause of obtaining uselessly wide bands. Unfortunately, we don't have the flexibility of narrowing these 
bands by restricting them to a fixed time interval $[0, T]$ of interest. The very reason that they are asymptotically distribution free is that they are valid asymptotically on the whole support.

In case of the constant width bootstrap band in (iii) and the standardized or 'equal precision' bootstrap band in (iv) we have the just mentioned flexibility, and working on a fixed interval $[0, T]$ requires less regularity conditions. The price we pay is that at each single application we have to conduct a bootstrap Monte Carlo simulation to approximate $c_{n}=c_{n}(\alpha, p, T)$ and $d_{n}=d_{n}(\alpha, p, T)$. Suppose we draw from $X_{1}, \ldots, X_{n}$ bootstrap samples $b$ times and accordingly compute both

$$
\Gamma_{n}=\sup _{0 \leq t \leq T} \sqrt{n}\left|\tilde{R}_{n}^{(p)}(t)-R_{n}^{(p)}(t)\right| \quad \text { and } \quad \Delta_{n}=\sup _{0 \leq t \leq T} \sqrt{n}\left|\frac{\tilde{R}_{n}^{(p)}(t)-R_{n}^{(p)}(t)}{\sigma_{n}(t)}\right|,
$$

$b$ times and denote the resulting ordered values by $\Gamma_{n}(1, b) \leq \cdots \leq \Gamma_{n}(b, b)$ and $\Delta_{n}(1, b) \leq \cdots \leq \Delta_{n}(b, b)$, respectively. Then, if $b$ is large enough, $\Gamma_{n}([(1-\alpha) b], b)$ and $\Delta_{n}([(1-\alpha) b], b]$ are the respective bootstrap approximations of $c_{n}$ and $d_{n}$, where $[\cdot]$ denotes integer part.

Following the strike example in Section 3, a small scale simulation study of actual coverage probabilities is reported in Section 4.

The problem of estimating percentile residual life from randomly censored data is considered by Chung (1986) and S. Csörgő (1987, 1988).

\section{Monte Carlo determination of $\lambda(p, \alpha)$}

If we generate the random numbers $U_{1}, \ldots, U_{k}$ uniformly distributed in $(0,1)$, consider their empirical distribution function $G_{k}(x)=k^{-1} \#\left\{1 \leq j \leq k: U_{j} \leq x\right\}$ and form the uniform empirical process $\alpha_{k}(x)=k^{1 / 2}\left(G_{k}(x)-x\right), 0 \leq x \leq 1$, then, since $\alpha_{k}(\cdot)$ converges weakly to $B(\cdot)$ as $k \rightarrow \infty$, the asymptotic distribution of

$$
A^{(p)}(k)=\sup _{0 \leq x \leq 1}\left|\alpha_{k}(1-p(1-x))-p \alpha_{k}(x)\right|
$$

is the unknown limiting distribution in (i) and (ii) of the theorem. Fixing a large enough sample size $k$ and repeating this operation $m$ times, the number of Monte Carlo trials, denote by $A_{\mathrm{l}, m}^{(p)}(k) \leq \cdots \leq A_{m, m}^{(p)}(k)$ the order statistics of the resulting realizations $A_{1}^{(p)}(k), \ldots, A_{m}^{(p)}(k)$. Then $A_{[(1-\alpha) m], m}^{(p)}(k)$ is the respective Monte Carlo approximation to $\lambda(p, \alpha)$.

As mentioned in Point (4) of Section 4 in Csörgő and Csörgő (1987), theoretical considerations suggest that the sample size $k=50$ should be sufficient. However, in order to be on the safe side, we have chosen and used $k=100$. Also, trying to provide the highest affordable protection against instability when simulating large quantiles belonging to $\alpha=0.01$, we have worked with the large trial size $m=5000$. The 21 rows of Table 1 contain our Monte Carlo values of $\lambda(p, \alpha)$ for the 21 selected values of $p$ as indicated and for 6 selected values of $\alpha$, leading to the most usual asymptotic coverage probabilities $1-\alpha$. 


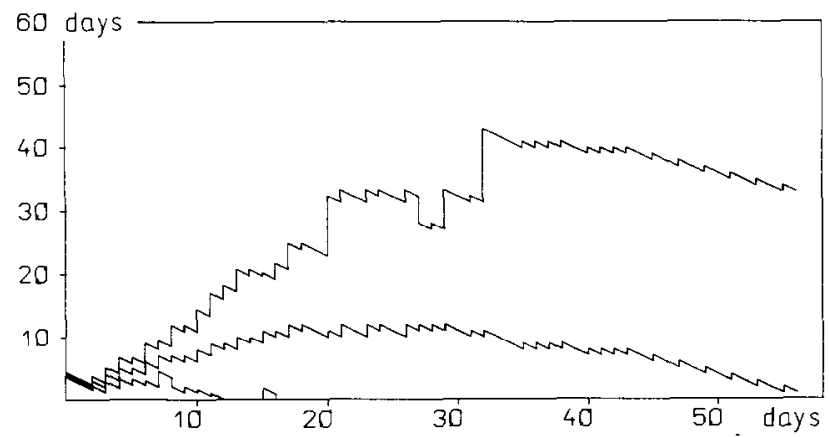

Fig. 3. The band of (i) corresponding to the solid-line histogram estimate in Fig. 2.

(1981)) records the durations of 840 strikes in the United Kingdom that commenced in 1965 and lasted longer than 1 day. Using these data, Csörgö and Csörgö (1987) have constructed the asymptotically $1-\alpha=0.9$ confidence band for the percentile function $R^{(1-p)}(3), 0.14 \leq p \leq 0.86$, of a strike lasting longer than 3 days. Here we construct bands for $R^{(1 / 2)}(t), t \geq 0$.

For the longest 69 strikes longer than 20 days the data are grouped by 5 days up to 50 days with remaining 8 strikes longer than 50 days. In the present study we have taken the liberty to redistribute these grouped data more or less uniformly within each group and assumed that the longest 8 strikes lasted for $51,52, \ldots, 58$ days. This arbitrary change is just to make the jump structure of $R^{(1 / 2)}(\cdot)$ and of the band contours somewhat finer, but the difference otherwise is hardly noticable.

For the bands in (i) and (iv) of the theorem we had to construct the corresponding density estimates. We have chosen histogram estimates, corresponding to the obvious choices of 1,2 and 3 days as cell widths, and kernel estimates

$$
f_{n}(x)=\frac{1}{n h_{n}} \sum_{j=1}^{n} K\left(\frac{x \quad X_{j}}{h_{n}}\right)
$$

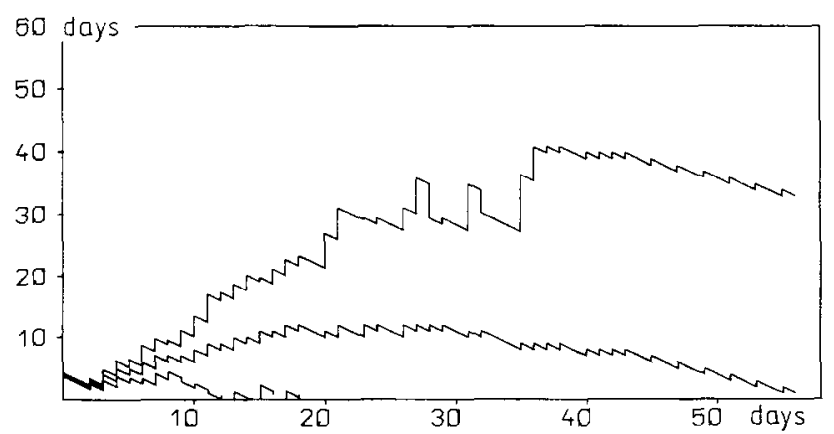

Fig. 4. The band of (i) corresponding to the dotted-line normal kernel estimate in Fig. 2. 


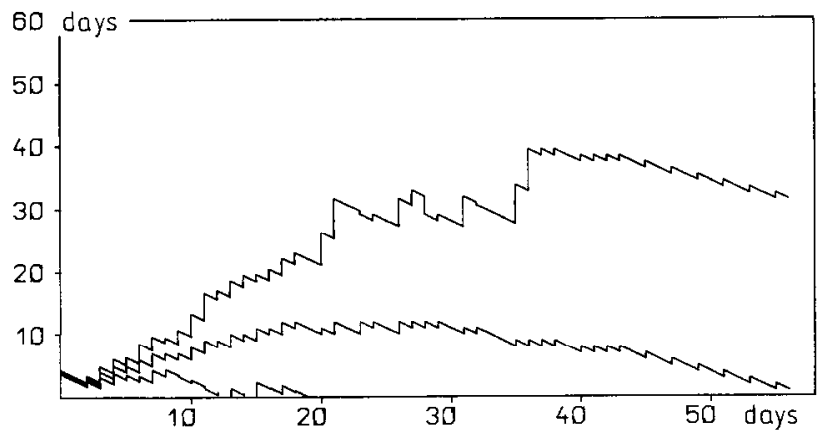

Fig. 5. The band of (i) corresponding to the broken-line Bartlett-Epanechnikov kernel estimate in Fig. 2.

corresponding to the standard normal density and to the Bartlett-Epanechnikov density

$$
K_{0}(y)= \begin{cases}\frac{3}{4}\left(1-y^{2}\right), & |y|<1, \\ 0, & |y| \geq 1,\end{cases}
$$

as kernels $K$, and in the latter varied the window width $h_{n}$ to obtain curves that match the histograms. Of course, the larger is $h_{n}$, the flatter and smoother are the kernel estimates. As a typical picture, Figure 2 gives the two-day histogram (solid line) and the virtually coinciding normal-kernel (with $h_{840}=0.6$; dotted line) and $K_{0}$-kernel (with $h_{480}=1.3$; broken line) density estimates.

Figures 3, 4 and 5 depict the three bands of (i) corresponding to the density estimates in Figure 2 with $\lambda(0.5,0.1)=1.1$ taken from Table 1 , all for the median residual duration $R^{(1 / 2)}(t), t \geq 0$, and for asymptotic coverage probability $1-\alpha=0.9$. The band of (ii) with the same parameters is contained in Figure 6 . The latter band cannot be drawn beyond 22 days because, in spite of the large sample size $n=840$, the argument of $Q_{840}$ in the upper contour reaches in fact 1 there. The middle graph is always the estimate $R_{840}^{(1 / 2)}(t), t \geq 0$, with $R_{840}^{(1 / 2)}(0)=4$ days estimating the median of

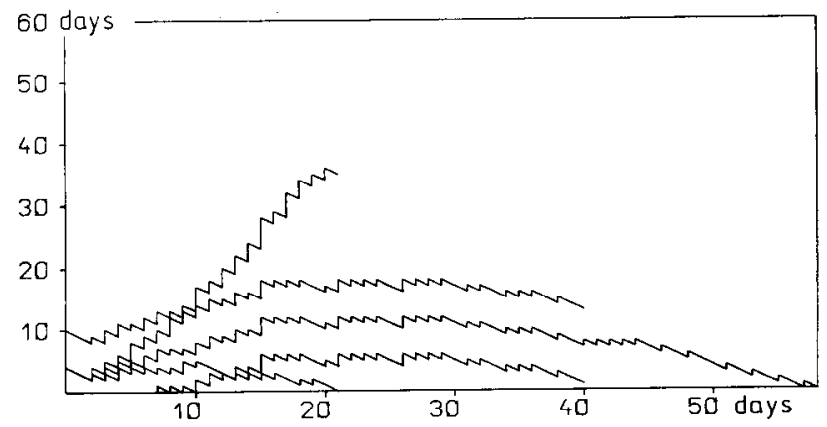

Fig. 6. The band of (ii) and the constant-width bootstrap band of (iii). 


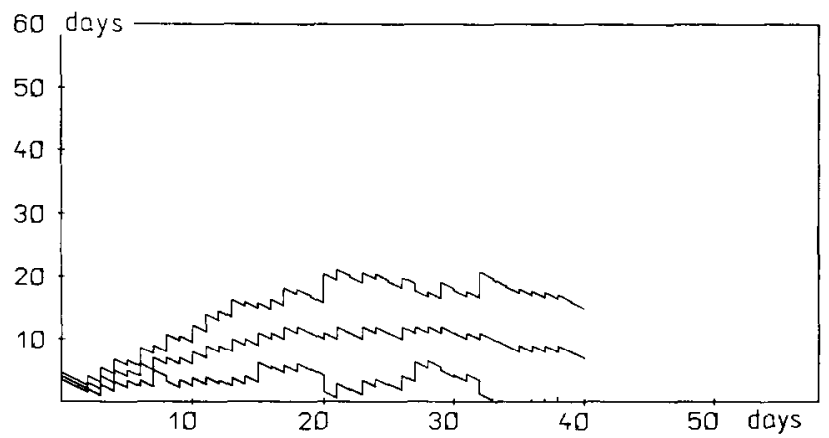

Fig. 7. The band of (iv) corresponding to the solid-line histogram estimate in Fig. 2.

the duration time distribution. In Figures 7,8 and 9 , the three standardized bootstrap bands of (iv) are drawn, corresponding to the density estimates in Figure 2, while the constant-width bootstrap band of (iii) is in Figure 6. All these are again for $R^{(1 / 2)}$ and $1-\alpha=0.9$. To illustrate the mentioned flexibility, for the four bootstrap bands we have chosen the interval $(0,40)$ to work on. All these four bands are based on $b=3000$ bootstrap replicas. While it is the constant width bootstrap band that looks the nicest here, it is probably always advantageous to draw all the bands to get a better feeling of the data at hand.

Many more figures and a more detailed discussion are given in Viharos (1988). Schmittlein and Morrison (1981) argue that the straight-line median residual life function $R^{(1 / 2)}(t)=\left(2^{1 / r}-1\right)(\alpha+t), t \geq 0$, of a second kind Pareto distribution function $F(t)=1-(\alpha /(\alpha+t))^{r}$ with estimated parameters $\alpha=\hat{\alpha}=4.94$ and $r=\hat{r}=1.83$, obtained by maximum likelihood, fits the data well. This straight line indeed appears to pick the increasing trend quite nicely up to about 20 days following the median duration. However, it fails to account for the well-known fact that strikes tend to grow tired, and after a certain turning point their tendency is to end sooner or later. Figures 2-9 suggest that for longer British strikes in 1965 this turning point

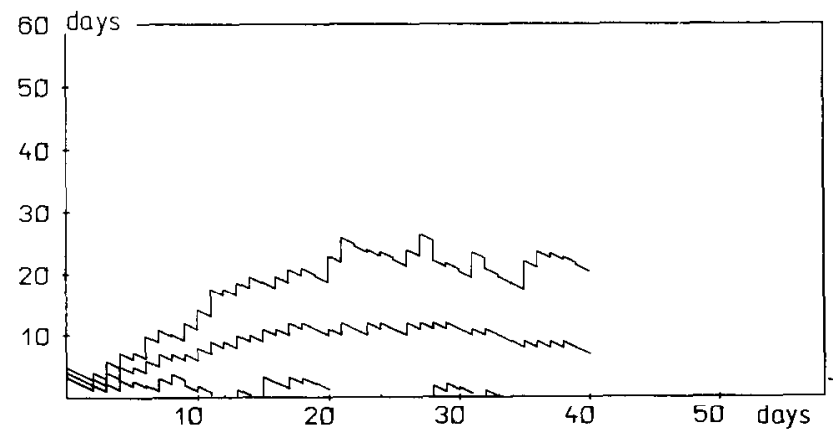

Fig. 8. The band of (iv) corresponding to the dotted-line normal kernel estimate in Fig. 2. 


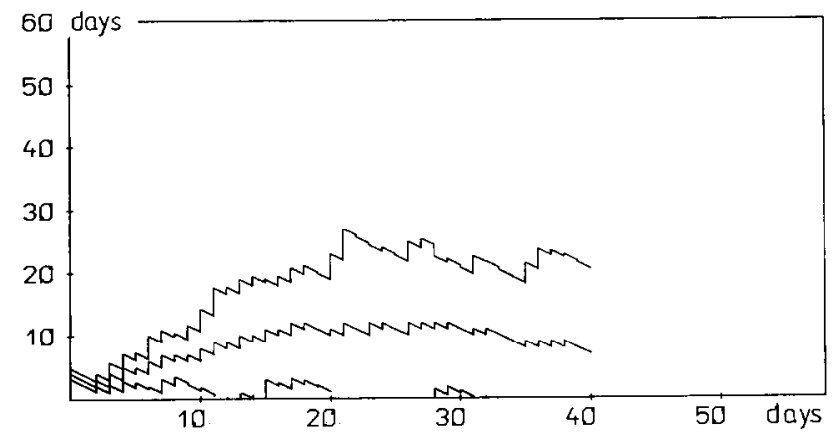

Fig. 9. The band of (iv) corresponding to the broken-line Bartlett-Epanechnikov kernel estimate in Fig. 2.

was somewhere between 15 and 30 days following the median duration of about 4 days.

\section{A simulation study of coverage probabilities}

For the standard exponential distribution with mean 1 and the standard Weibull distribution with shape parameter 2 , we conducted a small scale simulation study of the actual coverage probabilities of the bands in (ii) and (iii) when the asymptotic nominal coverage probability is $1-\alpha=0.9$. For both of the distributions the bootstrap band in (iii) was constructed on the interval $(0, Q(0.9))$, and for both distributions and both bands we used the sample sizes $n=50$ and 100 , while the bootstrap replica size $b$ for the band in (iii) was chosen to be $b=2 n$. Based on 500 Monte Carlo repetitions, the actual coverage of the bands in (ii) was between $90 \%$ and $92 \%$ in all four cases, though on intervals with average length considerably smaller than $Q(0.9)$. On the other hand, using the same 500 repetitions, the bootstrap bands of (iii) turned out to be very wide and conservative with actual coverage between $97 \%$ and $99 \%$ in all four cases. Hence our conclusion is that for the two distributions considered the sample size 100 and/or bootstrap replica size 200 are not enough to make the bootstrap bands work precisely. More details are again in Viharos (1988).

\section{References}

Chung, C.F. (1986). Confidence bands for quantile function and percentile residual lifetime under random censorship. Unpublished Ph.D. thesis, Cerleton University, Ottawa, Canada.

Csörgö, M. and S. Csörgö (1987). Estimation of percentile residual life. Oper. Res. 35, 598-606.

Csörgó, S. (1987). Estimating percentile residual life under random censorship. In: W. Sendler, Ed. Contributions to Stochastics, Physica-Verlag, Heidelberg, 19-27. 
Csörgö, S. (1988). Estimation in the proportional hazards model of random censorship, Statistics 19 , $437-463$.

Csörgö, S. and D.M. Mason (1989). Bootstrapping empirical functions. Ann. Statist. 17, 1447-1471.

Lancaster, T. (1972). A stochastic model for the duration of a strike. J. Roy. Statist. Soc. Ser. A 135, 257-271.

Rényi, A. (1970). Probability Theory. North-Holland, Amsterdam.

Schmittlein, D.C. and D.G. Morrison (1981). The median residual life time: a characterization theorem and an application. Oper. Res. 29, 392-399.

Viharos, L. (1988). Confidence bands for the percentile residual lifetime function. Students' Mathematical Workshop Notes, University of Szeged, Szeged, Hungary. [In Hungarian.] 



\title{
Fixed-width interval estimation of the minimum point of a regression function based on the Kiefer-Wolfowitz procedure
}

\author{
Marek Męczarski \\ Central School of Planning and Statistics, Institute of Econometrics, PL-02-554 Warsaw, Poland \\ Received 4 November 1988; revised manuscript received 20 January 1990 \\ Recommended by J. Koronacki
}

\begin{abstract}
A version of the central limit theorem for the Kiefer-Wolfowitz procedure is stated. One constructs an asymptotically consistent fixed-width confidence interval for the minimum of a regression function. It is shown that the first moment of the corresponding stopping rule is finite. Both the construction and properties of the estimates of unknown parameters and an adaptive version of the procedure are presented.
\end{abstract}

AMS Subject Classification: Primary 62L20; secondary 62L15, 62G05, $62 \mathrm{G} 15$.

Key words and phrases: Kiefer-Wolfowitz procedure; central limit theorem; sequential fixed-width interval estimation; asymptotic consistency; asymptotic efficiency; adaptive procedure.

\section{Introduction and assumptions}

Let us consider the family of real random variables $\{Y(x)\}_{x \in \mathbb{R}}$. We want to estimate a point $\theta \in \mathbb{R}$ such that the regression function $f(x)=E Y(x)$ attains its minimum at $\theta$. We will construct an asymptotic fixed-width confidence interval, i.e. two statistics $X_{n}$ and $N(d)$ such that

$$
\lim _{d \rightarrow 0} P\left(\left|X_{N(d)}-\theta\right| \leq d\right)=\eta
$$

for the fixcd number $\eta \in(0,1)$.

Let $X_{n}$ be given by the Kiefer-Wolfowitz (KW) procedure

$$
X_{n+1}=X_{n}-(A / n)\left(Y\left(X_{n}+c_{n}\right)-Y\left(X_{n}-c_{n}\right)\right) /\left(2 c_{n}\right), \quad n=1,2, \ldots,
$$

with $A>0, X_{1} \in \mathbb{R}$. Let $c_{n}=C n^{-\gamma}$ with $C>0$, and $\gamma \in\left(0, \frac{1}{4}\right)$. Let

$$
\varepsilon_{x} \stackrel{\text { def }}{=} Y(x)-f(x), \quad \mathscr{F}_{n} \stackrel{\text { def }}{=} \sigma\left(\varepsilon_{X_{i} \pm c_{i}}, \varepsilon_{X_{i}}, 1 \leq i \leq n\right) .
$$

Then $X_{n+1}$ is $\mathscr{F}_{n}$-measurable. 